\title{
Difference in pulmonary permeability between indirect and direct acute respiratory distress syndrome assessed by the transpulmonary thermodilution technique: a prospective, observational, multi-institutional study
}

Kenichiro Morisawa ${ }^{1 *}$, Shigeki Fujitani', Yasuhiko Taira' ${ }^{1}$, Shigeki Kushimoto ${ }^{2}$, Yasuhide Kitazawa $^{3}$, Kazuo Okuchi $^{4}$, Hiroyasu Ishikura ${ }^{5}$, Teruo Sakamoto ${ }^{6}$, Takashi Tagami ${ }^{7}$, Junko Yamaguchi ${ }^{8}$, Manabu Sugita ${ }^{9}$, Yoichi Kase $^{10}$, Takashi Kanemura $^{11}$, Hiroyuki Takahashi ${ }^{12}$, Yuuichi Kuroki ${ }^{13}$, Hiroo Izumino ${ }^{14}$, Hiroshi Rinka ${ }^{15}$, Ryutarou Seo ${ }^{16}$, Makoto Takatori ${ }^{17}$, Tadashi Kaneko ${ }^{18}$, Toshiaki Nakamura ${ }^{19}$, Takayuki Irahara ${ }^{20}$, Nobuyuki Saitou ${ }^{21}$, Akihiro Watanabe ${ }^{22}$ and PiCCO Pulmonary Edema Study Group

\footnotetext{
Abstract

Background: Acute respiratory distress syndrome (ARDS) is characterized by the increased pulmonary permeability secondary to diffuse alveolar inflammation and injuries of several origins. Especially, the distinction between a direct (pulmonary injury) and an indirect (extrapulmonary injury) lung injury etiology is gaining more attention as a means of better comprehending the pathophysiology of ARDS. However, there are few reports regarding the quantitative methods distinguishing the degree of pulmonary permeability between ARDS patients due to pulmonary injury and extrapulmonary injury.

Methods: A prospective, observational, multi-institutional study was performed in 23 intensive care units of academic tertiary referral hospitals throughout Japan. During a 2-year period, all consecutive ARDS-diagnosed adult patients requiring mechanical ventilation were collected in which three experts retrospectively determined the pathophysiological mechanisms leading to ARDS. Patients were classified into two groups: patients with ARDS triggered by extrapulmonary injury (ARDSexp) and those caused by pulmonary injury (ARDSp). The degree of pulmonary permeability using the transpulmonary thermodilution technique was obtained during the first three intensive care unit (ICU) days.

(Continued on next page)
}

\footnotetext{
*Correspondence: kmori0079@yahoo.co.jp

'Department of Emergency and Critical Care Medicine, St. Marianna University School of Medicine, 2-16-1, Sugao, Miyamae-ku, Kawasaki-shi, Kanagawa-ken 216-8511, Japan

Full list of author information is available at the end of the article
} 


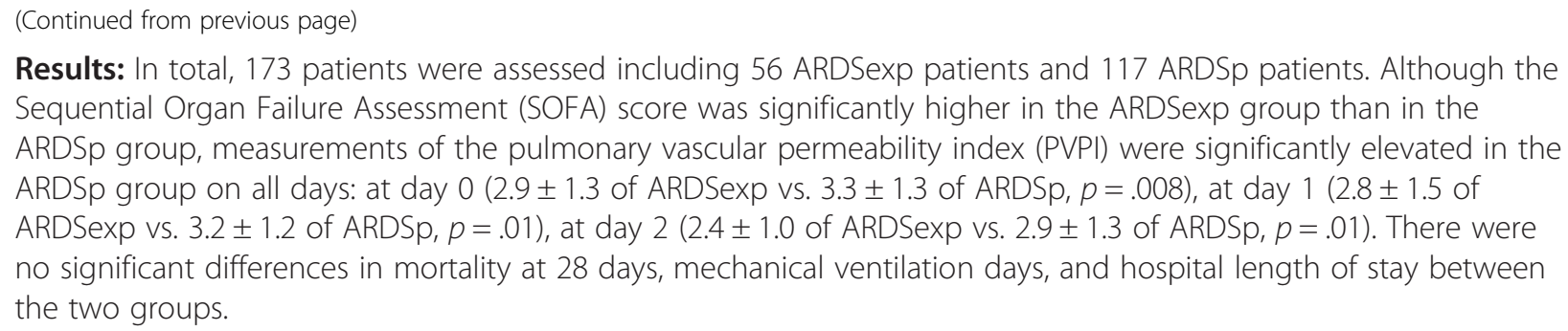

Conclusions: The results of this study suggest the existence of several differences in the increased degree of pulmonary permeability between patients with ARDSexp and ARDSp.

Trial registration: This report is a sub-group analysis of the study registered with UMIN-CTR (ID UMIN000003627).

Keywords: Acute pulmonary edema, Acute respiratory distress syndrome, Indirect injury, Direct injury, Pulmonary vascular permeability, Transpulmonary thermodilution technique

\section{Background}

Acute respiratory distress syndrome (ARDS) is a challenging disease renowned to any intensive care unit (ICU) and is associated with a high mortality rate, as documented by recent systematic reviews finding a pooled mortality of $44 \%$ and $43 \%[1,2]$.

ARDS is characterized by the increased pulmonary capillary permeability secondary to diffuse alveolar inflammation and injuries [3]. Common risk factors can be classified into two groups: extrapulmonary causes (indirect etiologies: ARDSexp) or pulmonary causes (direct etiologies: ARDSp). The major causes of ARDSexp include non-pulmonary sepsis, major trauma, pancreatitis, severe burns, non-cardiogenic shock, drug overdose, and multiple transfusions, while the main causes of ARDSp are pneumonia, aspiration of gastric contents, inhalation injury, pulmonary contusion, pulmonary vasculitis, and drowning [4]. Lung injuries of different origins may hold distinct pathophysiology, lung morphology, radiology, respiratory mechanics, and response to managements [5]. This distinction between an indirect and a direct etiology of lung injury is gaining more attention as a means of better comprehending the pathophysiology of ARDS and possibly for modifying ventilator management [6,7]. Several studies have reported the difference between respiratory mechanics and severity between ARDSexp and ARDSp [6-8]. Gattinoni et al. [6] measured the elastic properties of the lung and chest wall by using the quasistatic technique and reported that the differences in respiratory mechanics and response to positive endexpiratory pressure (PEEP) between patients with ARDSexp and those with ARDSp were predominantly due to collapse. On the other hand, a systematic review consisting of 34 studies exhibited no difference in mortality between both groups [9]. Presently, we believe that radiological imaging, respiratory mechanics, as well as response to PEEP features between both groups do not affect mortality. We consider that no difference in mortality between both groups may depend on the same therapeutic strategy given to all ARDS cases. ARDS patient outcomes may be improved with specific modified therapies that would address the underlying pathologies during the initial stage. We believe that more quantitative methods estimating the heterogeneity of ARDS at the initial stage and a customized therapy for each individual case may be helpful to appropriately manage ARDS patients.

There are few quantitative methods that distinguish between both ARDS categories during their initial stages. As ARDS is a syndrome and includes various clinical conditions, the absolute difference between ARDSexp and ARDSp is still unknown. We hypothesized that the degree of increased pulmonary permeability caused by several underlying diseases may be different between patients within the two ARDS categories. We set out to determine whether there were any differences between the two groups in terms of the degree of pulmonary edema during the initial ICU stages.

The transpulmonary thermodilution technique system (PiCCO ${ }^{\circ}$, Pulsion Medical Systems, Munich, Germany) is able to establish the degree of pulmonary vascular permeability with the pulmonary vascular permeability index (PVPI) [10]. Kirov et al. [11] reported that the value of PVPI was high in a sheep model with ARDS. Furthermore, a clinical study by Monnet et al. [12] showed that PVPI was higher in patients with ARDS than in those with cardiogenic pulmonary edema. These results suggest that PVPI seemed to be useful to estimate the difference in pulmonary vascular permeability between the types of pulmonary edema [13]. We previously reported that PVPI was significantly higher in ARDS patients than in patients who had cardiogenic pulmonary edema despite both having diffuse bilateral infiltrates of the lung and hypoxemia. A PVPI of 2.6-2.85 
was proposed as the differential value to distinguish 'real' ARDS with permeability edema from cardiogenic edema or atelectasis which could lead to clinical symptoms that mimic ARDS [14]. Therefore, PVPI might reflect the variety of difference of causes that lead to ARDS.

To our knowledge, little data regarding the difference of PVPI elevation between ARDSexp and ARDSp in the ICU setting is available. The aim of this study was to evaluate the difference in pulmonary vascular permeability, using the value of PVPI between patients with ARDSexp and those with ARDSp.

\section{Methods}

We undertook a prospective, observational, multi-institutional study from March 2009 to August 2011. Patients admitted to the ICU of 23 hospitals in different regions of Japan were screened for ARDS as a sub-analysis of a clinical observational study utilizing hemodynamic monitoring with $\mathrm{PiCCO}^{\circ}$ [14]. The study was approved by the institutional ethics committee, and written informed consent was obtained from all study participants. This study was also registered with the University Hospital Medical Information Network (UMIN) Clinical Trials Registry (UMINCTR ID UMIN000003627).

We assessed medical and surgical patients according to the following five criteria: (1) older than 15 years of age (no upper age limit), (2) acute respiratory insufficiency expected to require mechanical ventilatory support in ICU for at least $48 \mathrm{~h}$, (3) $\mathrm{PaO}_{2} / \mathrm{FiO}_{2}$ (P/F) ratio of $\leq 300 \mathrm{mmHg}$, (4) bilateral infiltrates on chest radiograph, (5) monitoring by the transpulmonary thermodilution technique as per the discretion of attending physicians. Patients were excluded if one of the following criteria was present: definition of ARDS over 5 days prior to eligibility; past history of chronic respiratory insufficiency (mainly, chronic obstructive pulmonary disease); post lung resection/pneumonectomy; pulmonary thromboembolism; severe peripheral arterial disease; cardiogenic shock (cardiac index $<1.5 \mathrm{~L} / \mathrm{min} / \mathrm{m}^{2}$ ); acute phase of trauma with lung contusion, drowning, and burns; unsuitable situation for monitoring with $\mathrm{PiCCO}^{\circ}$. Three or more experts (intensive care, respiratory, and cardiology) determined the diagnosis of ARDS taking into account medical history, clinical features involving computed tomography (CT) appearances, respiratory and hemodynamic variables, and clinical course with therapy. We excluded all cases of respiratory failure that were caused by massive pneumothorax, atelectasis, and pleural effusion. Any case with an extravascular lung water index (EVLWI) $<10 \mathrm{~mL} / \mathrm{kg}$ was excluded [14].

The following data were collected from the medical records: age, gender, underlying disease, hospital length of stay, length of mechanical ventilation, and clinical outcome at 28 days (survival or death). We also calculated the Acute Physiology and Chronic Health Evaluation (APACHE) II score, Sequential Organ Failure Assessment (SOFA) score, PEEP, and P/F ratio at day 0 . Once the above-mentioned criteria were fulfilled, we commenced $\mathrm{PiCCO}^{\circ}$ measurement data collection in which all patients were monitored for three consecutive days: day 0 , day 1 , and day 2. PVPI and extravascular lung water (EVLW) were measured concurrently. At the same time, intrathoracic blood volume (ITBV) was measured as volumetric parameters by $\mathrm{PiCCO}^{\circ}$. We calculated EVLWI with predicted body weight and intrathoracic blood volume index (ITBI) with area of body surface. We utilized predicted body weight to index EVLW and ITBV according to previous studies which determined that EVLWI indexed to the predictive body weight was a better prognostic indicator for ARDS patients than EVLWI indexed to the actual body weight $[15,16]$.

From the various diagnosed causes of ARDS, we classified all patients into two groups (indirect injury group or direct injury group). We assessed each patient's medical history, clinical presentation, chest CT, radiography, and echocardiography. Indirect injury group (ARDSexp) included patients with ARDS secondary to non-pulmonary sepsis, major trauma, pancreatitis, severe burns, noncardiogenic shock, drug overdose, and multiple transfusions. The direct injury group (ARDSp) included patients with ARDS caused by pneumonia, aspiration of gastric contents, inhalation injury, pulmonary contusion, pulmonary vasculitis, and drowning [4]. Patients that presented with acute phase of inhalation burn, pulmonary contusion, and drowning were excluded from the study. All the experts who conducted the classification were blinded to the value of PVPI.

\section{Statistical analysis}

Descriptive frequencies were expressed using the mean and standard deviation (SD). Categorical data was analyzed using Pearson's chi-square test for the characteristics and 28-day mortality. The independent $t$ test was used for normally distributed variables, or the MannWhitney $U$ test was used for non-normally distributed variables. All tests were two-sided, and $p<.05$ was considered to be significant. We analyzed all data using SPSS software, version 18 for Windows (SPSS, Chicago, IL, USA).

\section{Results}

During the study period from March 2009 to August 2011, we assessed a total of 301 patients that were subjected to mechanical ventilation. Of these, 94 were excluded due to reasons presented in Figure 1. Of the remaining 207 cases with absolute ARDS, the experts categorized 34 patients as neither ARDSexp nor ARDSp groups due to lack of a clear identification of the 


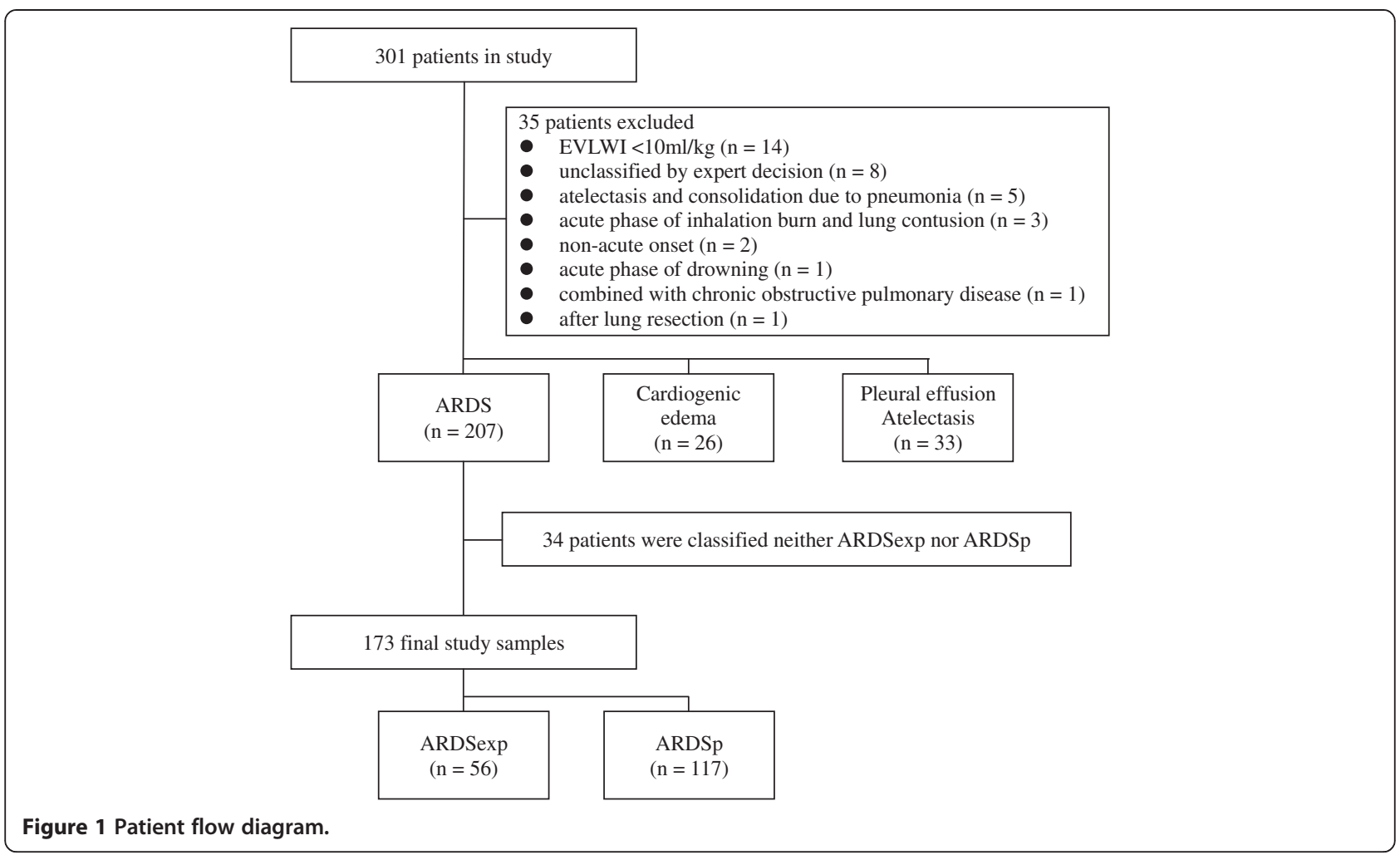

underlying cause or a mixed clinical presentation of the disease. The remaining 173 patients were included into the final analysis.

Patients' characteristics are summarized in Table 1. Of these, the age range of affected patients was 21-93 years (median 67 years), with 67\% males. Fifty-six patients (32\%) had a diagnosis of ARDSexp, and 117 patients (68\%) had a diagnosis of ARDSp. The most common cause of ARDSexp was sepsis (71\%) and pneumonia (80\%) in ARDSp. Significant differences in baseline characteristics were seen between the two groups with the SOFA score (ARDSexp $12 \pm 4$ vs. ARDSp $10 \pm 3 ; p=.0001$ ) and the concentration of serum albumin (2.4 g/dL of ARDSexp vs. $2.7 \mathrm{~g} / \mathrm{dL}$ of ARDSp; $p=.02$ ). There were no significant differences in APACHE II, PEEP, and mean airway pressure initially. The $\mathrm{P} / \mathrm{F}$ ratio tended to be lower in ARDSp than ARDSexp (163 \pm 73 of ARDSexp vs. $143 \pm$ 70 of ARDSp; $p=.09$ ).

Results of measurements of $\mathrm{PiCCO}^{\circ}$ at day 0 , day 1 , and day 2 are summarized in Table 2 . The measurement of PVPI (Figure 2) was significantly elevated in ARDSp compared with ARDSexp on all days: at day $0(2.9 \pm 1.3$ of ARDSexp vs. $3.3 \pm 1.3$ of ARDSp, $p=.008$ ), at day 1 ( $2.8 \pm 1.5$ of ARDSexp vs. $3.2 \pm 1.2$ of ARDSp, $p=.01)$, at day $2(2.4 \pm 1.0$ of ARDSexp vs. $2.9 \pm 1.3$ of ARDSp, $p=.01$ ). All ITBI data from both groups was in the upper range of normal. Although there was no significant difference in the EVLWI at day 0 and day 1, the level of EVLWI in the ARDSp group was significantly higher than that in the ARDSexp patients at day $2(14.9 \pm 6.0$ of ARDSexp vs. $17.6 \pm 7.8$ of ARDSp, $p=.02$ ).

Patients' outcomes are summarized in Table 3. There was no significant difference in 28-day mortality, hospital stay days, and ventilation days between the two groups as follows: mechanical ventilation (16 \pm 19 days of ARDSexp vs. $13 \pm 9$ days of ARDSp, $p=.85$ ), hospital length of stay ( $42 \pm 55$ days of ARDSexp vs. $40 \pm 56$ days of ARDSp, $p=.51$ ), and mortality at 28 days (32\% of ARDSexp vs. $44 \%$ of ARDSp, $p=.15$ ).

\section{Discussion}

ARDS is considered to be an expression of a diffuse inflammatory reaction within the lungs caused by a number of underlying diseases, which have a variety of pathophysiological courses being either of pulmonary or extrapulmonary origin. Thus, depending on its etiology, ARDS may present with obvious distinct characteristics. In this study, we attempted to establish the quantitative difference in pulmonary permeability between ARDSexp and ARDSp with PVPI obtained by the transpulmonary thermodilution technique $\left(\mathrm{PiCCO}^{\circ}\right)$.

The characteristics and clinical outcomes in both groups were similar to previous studies $[6,8,17,18]$. The prevalence of ARDSp in this study was higher compared 
Table 1 Characteristics of 173 patients at the initial measurement of thermodilution technique

\begin{tabular}{|c|c|c|c|}
\hline & $\begin{array}{c}\text { ARDSexp } \\
n=56\end{array}$ & $\begin{array}{l}\text { ARDSp } \\
n=117\end{array}$ & $p$ value \\
\hline Age (year), median & 65 & 72 & .03 \\
\hline Male, $n(\%)$ & $32(57)$ & $84(72)$ & .05 \\
\hline \multicolumn{4}{|l|}{ Causes of ARDS, $n(\%)$} \\
\hline Sepsis & $40(71)$ & & \\
\hline Trauma and burn & $10(18)$ & & \\
\hline Pancreatitis & $4(7)$ & & \\
\hline TRALI & $2(4)$ & & \\
\hline Pneumonia & & $94(80)$ & \\
\hline Aspiration & & $20(17)$ & \\
\hline Lung contusion & & $2(2)$ & \\
\hline Inhalation burn & & $1(1)$ & \\
\hline APACHE II score & $24 \pm 9$ & $23 \pm 8$ & .39 \\
\hline SOFA score & $12 \pm 4$ & $10 \pm 3$ & .0001 \\
\hline PEEP $\left(\mathrm{cmH}_{2} \mathrm{O}\right)$ & $9 \pm 5$ & $9 \pm 5$ & .36 \\
\hline $\mathrm{PaO}_{2} / \mathrm{FiO}_{2}$ ratio & $163 \pm 73$ & $143 \pm 70$ & .09 \\
\hline Mean airway pressure $\left(\mathrm{cmH}_{2} \mathrm{O}\right)$ & $17 \pm 6$ & $17 \pm 4$ & .55 \\
\hline $\mathrm{Na}(\mathrm{mmol} / \mathrm{L})$ & $135 \pm 19$ & $138 \pm 7$ & .37 \\
\hline $\mathrm{K}(\mathrm{mmol} / \mathrm{L})$ & $4.1 \pm 0.8$ & $4.0 \pm 0.9$ & .15 \\
\hline Albumin (g/dL) & $2.4 \pm 0.5$ & $2.6 \pm 0.8$ & .02 \\
\hline Glucose (mg/dL) & $159 \pm 61$ & $165 \pm 83$ & .95 \\
\hline Blood urea nitrogen $(\mathrm{mg} / \mathrm{dL})$ & $38 \pm 28$ & $36 \pm 25$ & .84 \\
\hline
\end{tabular}

Values are given as mean \pm standard deviation, unless otherwise indicated. ARDS acute respiratory distress syndrome, ARDSexp acute respiratory distress syndrome secondary to extrapulmonary cause, ARDSp acute respiratory distress syndrome secondary to pulmonary cause, TRALI transfusion-related acute lung injury, APACHE Acute Physiologic and Chronic Health Evaluation, SOFA Sequential Organ Failure Assessment, PEEP positive end-expiratory pressure.

to that of the ARDSexp group which mainly consisted of patients with sepsis and trauma, as opposed to ARDSp which consisted of those with pneumonia and aspiration $[6,8,17,18]$. Although these various clinical backgrounds may influence ARDS outcomes, no significant differences in 28-day mortality, length of hospital stay, and number of mechanical ventilation days were noted between the ARDSexp or ARDSp patients in this study. These results concur with a previously reported metaanalysis which manifested no difference in mortality between ARDSexp and ARDSp [9]. Agarwal et al. [8] also reported that categories of underlying disease did not affect the duration of hospitalization and the number of mechanical ventilation days, although this study showed that the initial severity of respiratory failure, $\mathrm{PaO}_{2} / \mathrm{FiO}_{2}$ ratio, was worse in patients with ARDSp than in those with ARDSexp. Each of the two ARDS groups characterized by different origins arguably has a different pathophysiological course for the lung permeability along with
Table 2 Results of the measurement by thermodilution technique

\begin{tabular}{cccc}
\hline & $\begin{array}{c}\text { ARDSexp } \\
\boldsymbol{n}=\mathbf{5 6}\end{array}$ & $\begin{array}{c}\text { ARDSp } \\
\boldsymbol{n}=\mathbf{1 1 7}\end{array}$ & $\boldsymbol{p}$ value \\
\hline Day 0 & & & \\
ITBI & $1,067 \pm 236$ & $1,015 \pm 270$ & .09 \\
EVLWI & $17.8 \pm 6.6$ & $19.0 \pm 7.1$ & .20 \\
PVPI & $2.9 \pm 1.3$ & $3.3 \pm 1.3$ & .008 \\
Day 1 & & & \\
ITB| & $1,069 \pm 309$ & $1,019 \pm 279$ & .37 \\
EVLWI & $17.0 \pm 8.0$ & $18.0 \pm 7.0$ & .16 \\
PVPI & $2.8 \pm 1.5$ & $3.2 \pm 1.2$ & .01 \\
Day 2 & & & .41 \\
ITBI & $1,095 \pm 263$ & $1,078 \pm 313$ & .02 \\
EVLWI & $14.9 \pm 6.0$ & $17.6 \pm 7.8$ & .01 \\
PVPI & $2.4 \pm 1.0$ & $2.9 \pm 1.3$ & \\
\hline
\end{tabular}

Values are given as mean \pm standard deviation. ARDSexp acute respiratory distress syndrome secondary to extrapulmonary cause, ARDSp acute respiratory distress syndrome secondary to pulmonary cause, ITBI intrathoracic blood volume index, EVLWI extravascular lung water index, PVPI pulmonary vascular permeability index.

similar clinical syndromes and outcomes. Hence, the initial separation of ARDSexp and ARDSp appears to be futile for predicting outcome from ARDS.

The value of ITBI as volumetric parameters by $\mathrm{PiCCO}^{\circ}$ in both groups was in the upper normal range. All participants were managed with appropriate fluid therapy during their admission to ICU. On the other hand, the value of PVPI was extremely elevated above the normal range (PVPI of 2.6-2.85; differential value to diagnose real ARDS [14]). We believe that these results suggest that all the included cases demonstrated permeability pulmonary edema, namely real ARDS with no cases of cardiogenic pulmonary edema or fluid overload which could be one of the most important biases to assess permeability pulmonary edema.

Patients with ARDSexp had significantly higher SOFA scores and lower albumin concentrations similarly to the report by Agarwal et al. [8]. We found PVPI in the ARDSexp group to be lower than that in those with ARDSp and speculate that the direct insult (i.e., caused by pulmonary infection, aspiration, and trauma) may have influenced the pulmonary permeability to a greater extent than the indirect insult with systematic hyper-cytokine storm caused by sepsis. We also noted EVLWI in the ARDSexp group to be lower than that in the ARDSp group. Generally, lower albumin concentrations lead to lower osmotic pressure, so that patients with hypoalbuminemia suffer from whole body edema, ascites, and especially pericardial effusion. Nevertheless, we found that direct insult caused the increase of EVLWI greater than systematic inflammation and hypoalbuminemia. 


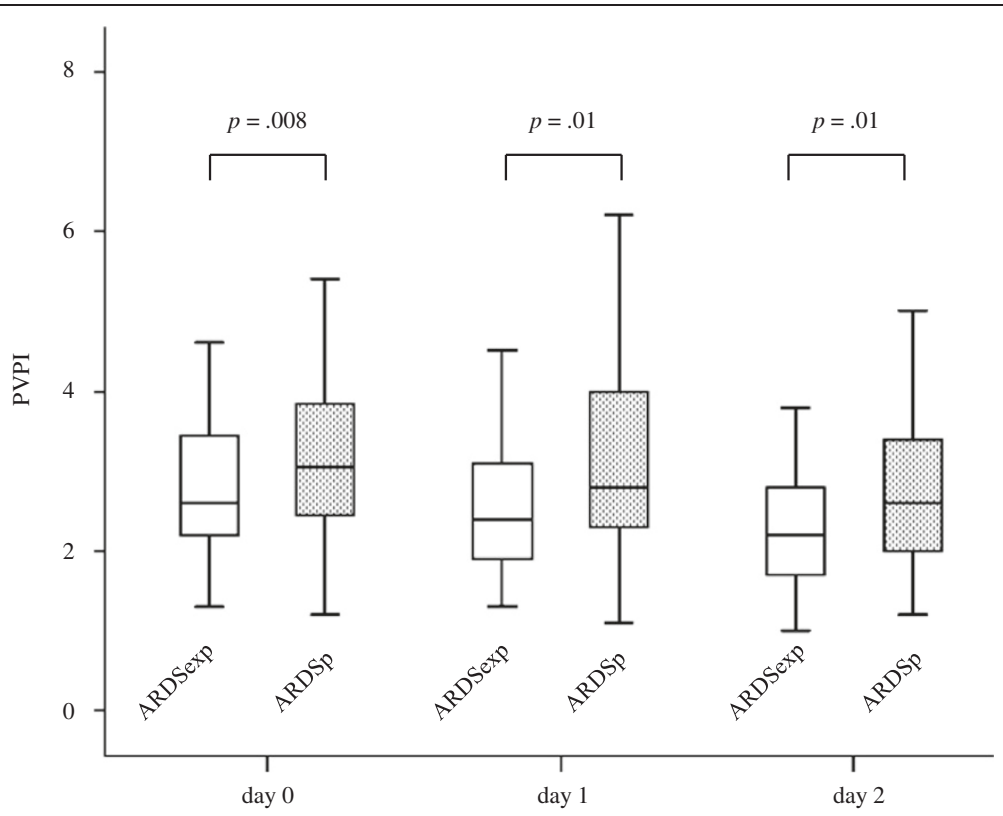

Figure 2 Comparison of PVPI between ARDSexp and ARDSp during the study period. The measurement of PVPI was significantly elevated in ARDSp compared with ARDSexp on all days: at day 0 ( $2.9 \pm 1.3$ of ARDSexp vs. $3.3 \pm 1.3$ of ARDSp, $p=.008$ ), at day 1 ( $2.8 \pm 1.5$ of ARDSexp vs. $3.2 \pm 1.2$ of ARDSp, $p=.01$ ), at day 2 (2.4 \pm 1.0 of ARDSexp vs. $2.9 \pm 1.3$ of ARDSp, $p=.01$ ).

This result could correspond to the animal report which compared the response of pulmonary epithelium damage caused by intraperitoneally or intratracheally exposed lipopolysaccharide and which concluded that the intratracheal insult leads to greater lung inflammation and ultrastructural morphologic changes. The levels of inflammatory cytokines (interleukin-6, interleukin-8 function homolog, and interleukin-10) found in the bronchoalveolar lavage fluid were significantly elevated in pulmonary ARDS models compared to extrapulmonary ARDS models, whereas no differences were observed in the number of infiltrating neutrophils [19]. This suggests, therefore, that the level of inflammation and the activation of neutrophils in patients with ARDSp may be higher than those in patients with ARDSexp.

There are several limitations to this study. First, the sample size was relatively small due to strict exclusion criteria to omit cases with complications that might also lead to hypoxemia and bilateral infiltrate on chest X-ray, including massive pneumothorax, atelectasis, and pleural effusion, to ensure that we included real ARDS patients.

Table 3 Clinical outcomes for participants

\begin{tabular}{lccc}
\hline & $\begin{array}{c}\text { ARDSexp } \\
\boldsymbol{n = 5 6}\end{array}$ & $\begin{array}{c}\text { ARDSp } \\
\boldsymbol{n = 1 1 7}\end{array}$ & $\boldsymbol{p}$ value \\
\hline 28-day mortality, $n$ (\%) & $18(32)$ & $51(44)$ & .15 \\
Hospital stay days & $42 \pm 55$ & $40 \pm 56$ & .51 \\
Ventilation days & $16 \pm 19$ & $13 \pm 9$ & .85 \\
\hline
\end{tabular}

Values are given as mean \pm standard deviation, unless otherwise indicated.
Second, the numbers of ARDSexp were fewer than those of ARDSp (56 of ARDSexp vs. 117 of ARDSp). There might be some statistical bias. We also excluded 94 cases with acute respiratory failure resulting from cardiogenic pulmonary edema, plural effusion, and atelectasis that might resemble ARDS (i.e., hypoxia with diffuse bilateral infiltrates) despite the lack of increased pulmonary permeability. We are concerned that our strict exclusion criteria may have excluded those with severe respiratory failure, and participants were limited to more moderate cases. However, we believe that our exclusion criteria provided an appropriate estimate of the key features of ARDS, namely participants with increased pulmonary permeability edema. We also excluded cases suspected of having both etiologies (ARDSexp and ARDSp) from our analysis. Finally, the varied and complicated factors of the underlying disease might influence the accuracy of thermodilution technique measurements. For this matter, we did not compare all those factors that could affect the accuracy of $\mathrm{PiCCO}^{\circ}$ findings but rather only the cases that were deemed possible of adequate $\mathrm{PiCCO}^{\circ}$ monitoring.

To our knowledge, there are few reports considering quantitative methods distinguishing both types of ARDS within the ICU setting. In a clinical situation, timely recognition of the difference and course in an ARDS lung is essential to drive and inform the clinical decision processes and dictate specific therapeutic strategies. The quantitative differences in EVLWI and PVPI obtained by the $\mathrm{PiCCO}^{\circ}$ system may be one important index that 
indicates a pathological difference between ARDSexp and ARDSp by estimating the amount of pulmonary permeability. Monitoring with $\mathrm{PiCCO}^{\circ}$ might give us timely quantitative information to make more informed clinical decisions.

\section{Conclusions}

The results of this study suggest that the $\mathrm{PiCCO}^{\circ}$ monitoring could be useful to distinguish between the state of pulmonary permeability in ARDSexp and ARDSp patients. We believe that there is a fundamental difference in the pathophysiology between these ARDSexp and ARDSp groups. Utilizing PVPI may provide us with timely quantitative information of pulmonary permeability in these two forms of ARDS. Additional studies should be undertaken in order to determine whether the clinical strategy guided with PVPI can have an impact on outcome and improve hypoxemia for patients with ARDS.

\section{Abbreviations}

APACHE II: Acute Physiology and Chronic Health Evaluation II; ARDS: acute respiratory distress syndrome; ARDSexp: ARDS triggered by extrapulmonary injury; ARDSp: ARDS triggered by pulmonary injury; CT: computed tomography; EVLW: extravascular lung water; EVLWl: extravascular lung water index; ICU: intensive care unit; ITBI: intrathoracic blood volume index; P/F ratio: $\mathrm{PaO}_{2} / \mathrm{FiO}_{2}$ ratio; PEEP: positive end-expiratory pressure; PVPI: pulmonary vascular permeability index; SOFA: Sequential Organ Failure Assessment.

\section{Competing interests}

$\mathrm{YT}$ is a member of the medical advisory board of Pulsion Medical Systems. $\Pi$, NS, and SK received honoraria for educational lectures from academia and Tokibo Co., Ltd (import trader of the PiCCO system) in scientific meetings. The remaining authors declare that they have no competing interests.

\section{Authors' contributions}

KM conducted the conception of this study, participated in the collection and analysis of data, and drafted the manuscript. All other authors participated in the collection and evaluation of data. All authors read and approved the final manuscript.

\section{Acknowledgements}

The authors would like to thank Lohman D. Brandon, MD, PhD, Department of Radiology, St. Marianna University School of Medicine, for his valuable contribution and advice in editing the present manuscript.

\section{Author details}

${ }^{1}$ Department of Emergency and Critical Care Medicine, St. Marianna University School of Medicine, 2-16-1, Sugao, Miyamae-ku, Kawasaki-shi, Kanagawa-ken 216-8511, Japan. ${ }^{2}$ Division of Emergency Medicine, Tohoku University Graduate School of Medicine, Sendai, Miyagi 980-8574, Japan. ${ }^{3}$ Critical Care Medical Center, Kinki University Hospital, Osakasayama, Osaka 589-8511, Japan. ${ }^{4}$ Advanced Emergency and Critical Care Center, Nara Medical University Hospital, Kashihara, Nara 634-8521, Japan. ${ }^{5}$ Department of Emergency and Critical Care Medicine, Fukuoka University Hospital, Fukuoka, Fukuoka 814-0180, Japan. ${ }^{6}$ Advanced Emergency and Critical Care Center, Kurume University Hospital, Kurume, Fukuoka 830-0011, Japan. ${ }^{7}$ Department of Emergency and Critical Care Medicine, Aidu Chuo Hospital, Aiduwakamatsu, Fukushima 965-8611, Japan. ${ }^{8}$ Department of Emergency and Critical Care Medicine, Nihon University School of Medicine Itabashi Hospital, Itabashi, Tokyo 173-8610, Japan. ${ }^{9}$ Department of Emergency and Critical Care Medicine, Juntendo University Nerima Hospital, Nerima, Tokyo 117-8521, Japan. ${ }^{10}$ Critical Care Medicine, Jikei University School of Medicine, Minato, Tokyo 105-0003, Japan. " Emergency and Critical Care Medicine, National Hospital Organization Disaster Medical Center, Tachikawa, Tokyo 190-0014, Japan. ${ }^{12}$ Department of Intensive Care Medicine, Saiseikai Yokohamashi Tobu
Hospital, Yokohama-shi, Kanagawa 230-8765, Japan. ${ }^{13}$ Department of Emergency and Critical Care Medicine, Social Insurance Chukyo Hospital, Nagoya, Aichi 457-0866, Japan. ${ }^{14}$ Emergency Medical Center, Nagasaki University Hospital, Nagasaki, Nagasaki 852-8102, Japan. ${ }^{15}$ Emergency and Critical Care Medical Center, Osaka City General Hospital, Miyakohuma, Osaka 534-0021, Japan. ${ }^{16}$ Intensive Care Unit, Kobe City Medical Center General Hospital, Kobe City, Hyogo 650-0047, Japan. ${ }^{17}$ Department of Anesthesia and Intensive Care, Hiroshima City Hospital, Hiroshima, Hiroshima 730-8518, Japan. ${ }^{18}$ Advanced Medical Emergency and Critical Care Center, Yamaguchi University Hospital, Ube City, Yamaguchi 755-8505, Japan. ${ }^{19}$ Intensive Care Unit, Nagasaki University Hospital, Nagasaki, Nagasaki 852-8102, Japan. ${ }^{20}$ Department of Emergency and Critical Care Medicine, Nippon Medical School Tama Nagayama Hospital, Tama-shi, Tokyo 206-8512, Japan.

${ }^{21}$ Department of Emergency and Critical Care Medicine, Nippon Medical School Chiba Hokusou Hospital, Inzai-shi, Chiba 270-1694, Japan.

${ }^{22}$ Department of Emergency and Critical Care Medicine, Nippon Medical School, Bunkyo-ku, Tokyo 113-8603, Japan.

Received: 23 November 2013 Accepted: 6 March 2014

Published: 25 March 2014

\section{References}

1. Phua J, Badia JR, Adhikari NK, Friedrich JO, Fowler RA, Singh JM, Scales DC, Stather DR, Li A, Jones A, Gattas DJ, Hallett D, Tomlinson G, Stewart TE, Ferguson ND: Has mortality from acute respiratory distress syndrome decreased over time?: a systematic review. Am J Respir Crit Care Med 2009, 179:220-227.

2. Zambon $M$, Vincent $J$ : Mortality rates for patients with acute lung injury/ ARDS have decreased over time. Chest 2008, 133:1120-1127.

3. Leaver SK, Evans TW: Acute respiratory distress syndrome. BMJ 2007 335:389-394.

4. Ranieri VM, Rubenfeld GD, Thompson BT, Ferguson ND, Caldwell E, Fan E, Camporota L, Slutsky AS: Acute respiratory distress syndrome: the Berlin definition. JAMA 2012, 307:2526-2533.

5. Pelosi P, Caironi P, Gattinoni L: Pulmonary and extrapulmonary forms of acute respiratory distress syndrome. Semin Respir Crit Care Med 2001, 22:259-268.

6. Gattinoni L, Pelosi P, Suter PM, Pedoto A, Vercesi P, Lissoni A: Acute respiratory distress syndrome caused by pulmonary and extrapulmonary disease. Different syndromes? Am J Respir Crit Care Med 1998, 158:3-11.

7. Pelosi P, D'Onofrio D, Chiumello D, Paolo S, Chiara G, Capelozzi VL, Barbas CS, Chiaranda M, Gattinoni L: Pulmonary and extrapulmonary acute respiratory distress syndrome are different. Eur Respir J Supp/ 2003, 42:48s-56s.

8. Agarwal R, Aggarwal AN, Gupta D, Behera D, Jindal SK: Etiology and outcomes of pulmonary and extrapulmonary acute lung injury/ARDS in a respiratory ICU in North India. Chest 2006, 130:724-729.

9. Agarwal R, Srinivas R, Nath A, Jindal SK: Is the mortality higher in the pulmonary vs the extrapulmonary ARDS? A meta analysis. Chest 2008, 133:1463-1473.

10. Michard F: Bedside assessment of extravascular lung water by dilution methods: temptations and pitfalls. Crit Care Med 2007, 35:1186-1192.

11. Kirov MY, Kuzkov W, Kuklin VN, Waerhaug K, Bjertnaes LJ: Extravascular lung water assessed by transpulmonary single thermodilution and postmortem gravimetry in sheep. Crit Care 2004, 8:R451-R458.

12. Monnet X, Anguel N, Osman D, Hamzaoui O, Richard C, Teboul J: Assessing pulmonary permeability by transpulmonary thermodilution allows differentiation of hydrostatic pulmonary edema from ALI/ARDS. Intensive Care Med 2007, 33:448-453.

13. Tagami T, Kushimoto S, Tosa R, Omura M, Yonezawa K, Akiyama G, Hirama $\mathrm{H}$, Yokota H: Plasma neutrophil elastase correlates with pulmonary vascular permeability: a prospective observational study in patients with pneumonia. Respirology 2011, 16:953-958.

14. Kushimoto S, Taira Y, Kitazawa Y, Okuchi K, Sakamoto T, Ishikura H, Endo T, Yamanouchi S, Tagami T, Yamaguchi J, Yoshikawa K, Sugita M, Kase Y, Kanemura T, Takahashi H, Kuroki Y, Izumino H, Rinka H, Seo R, Takatori M, Kaneko T, Nakamura T, Irahara T, Saito N, Watanabe A, The PiCCO Pulmonary Edema Study Group: The clinical usefulness of extravascular lung water and pulmonary vascular permeability index to diagnose and characterize pulmonary edema: a prospective multicenter study on the 
quantitative differential diagnostic definition for acute lung injury/acute respiratory distress syndrome. Crit Care 2012, 16:R232.

15. Phillips CR, Chesnutt MS, Smith SM: Extravascular lung water in sepsisassociated acute respiratory distress syndrome: indexing with predicted body weight improves correlation with severity of illness and survival. Crit Care Med 2008, 36:69-73.

16. Craig TR, Duffy MJ, Shyamsundar M, McDowell C, McLaughlin B, Elborn JS, McAuley DF: Extravascular lung water indexed to predicted body weight is a novel predictor of intensive care unit mortality in patients with acute lung injury. Crit Care Med 2010, 38:114-120.

17. Desai SR, Wells AU, Suntharalingam G, Rubens MB, Evans TW, Hansell DM: Acute respiratory distress syndrome caused by pulmonary and extrapulmonary injury: a comparative CT study. Radiology 2001, 218:689-693.

18. Brun-Buisson C, Minelli C, Bertolini G, Brazzi L, Pimentel J, Lewandowski K, Bion J, Romand JA, Villar J, Thorsteinsson A, Damas P, Armaganidis A, Lemaire F, ALIVE Study Group: Epidemiology and outcome of acute lung injury in European intensive care units. Results from the ALIVE study. Intensive Care Med 2004, 30:51-61.

19. Menezes SL, Bozza PT, Neto HC, Laranjeira AP, Negri EM, Capelozzi VL, Zin WA, Rocco PR: Pulmonary and extrapulmonary acute lung injury: inflammatory and ultrastructural analyses. J Appl Physiol 2005, 98:1777-1783.

doi:10.1186/2052-0492-2-24

Cite this article as: Morisawa et al.: Difference in pulmonary permeability between indirect and direct acute respiratory distress syndrome assessed by the transpulmonary thermodilution technique: a prospective, observational, multi-institutional study. Journal of Intensive Care 2014 2:24

\section{Submit your next manuscript to BioMed Central and take full advantage of:}

- Convenient online submission

- Thorough peer review

- No space constraints or color figure charges

- Immediate publication on acceptance

- Inclusion in PubMed, CAS, Scopus and Google Scholar

- Research which is freely available for redistribution 